\title{
Atividade Física em Pacientes Coronariopatas
} Physical Activity in Patients With Coronary Artery Disease

\section{RESUMO}

Objetivo: verificar a prevalência da atividade física em pacientes coronariopatas. Material e Métodos: Estudo transversal, de caráter descritivo, realizado em pacientes coronariopatas de um hospital do nordeste brasileiro. Investigou-se os dados sociodemográficos (idade, sexo, estado conjugal, escolaridade), estilo de vida (dieta, tabagismo, etilismo; hipercolesterolemia, diabetes, obesidade) e medidas antropométricas (peso, altura, índice de massa corporal, pressão arterial e circunferência abdominal). O nível de atividade física foi avaliado pelo Questionário Internacional de Atividade Física (IPAQ) versão longa, entre outubro a dezembro de 2014. Para verificar associação entre as variáveis foi utilizada a regressão logística univariada e multivariada e o testequi-quadrado de independência. Resultados: Dez $(53,0 \%)$ dos participantes foram considerados ativos pela avaliação das atividades consideradas habituais (doméstica ou laboral). As modalidades mais frequentes foram as atividades diárias como cuidar da casa $15(78 \%)$, atividade física como meio de transporte-bicicleta $7(36,8 \%)$ e atividade física no trabalho $5(26,3 \%)$. A prática de atividade como exercício físico (caminhada ou prática desportiva)não foi frequente na rotina dos coronariopatas $6(31,6 \%)$. Foi encontrada uma associação significativa entre o estado civil e a atividade física. Conclusão: As modalidades de atividades mais frequentes foram cuidar da casa, transporte-bicicleta, exercício ou recreação e no trabalho. Houve predomínio de indivíduos ativos nas atividades consideradas habituais moderadas em casa ou no trabalho. Foi encontrada uma associação significativa do nível de atividade e o estado civil.

\section{DESCRITORES}

Perfil de Saúde.Atividade Física.Doença das Coronárias.

\begin{abstract}
Objective: To determine the prevalence of physical activity in patients with coronary artery disease. Material and Methods: This was a cross-sectional, descriptive study with patients with coronary artery disease admitted to a hospital in northeastern Brazil. Socio-demographic data (age, sex, marital status, education), as well as lifestyle (diet, smoking, alcoholism; hypercholesterolemia, diabetes, obesity) and anthropometric measurements (weight, height, body mass index, blood pressure and waist circumference), were investigated. The level of physical activity was assessed by the International Physical Activity Questionnaire (IPAQ) Long version between October and December 2014. Univariate and Multivariate Logistic Regression and Chi-square test of independence were employed to check for association between variables. Results: Ten participants $(53.0 \%)$ were considered active in the evaluation of usual activities either housekeeping or labor-related. The most frequent modalitieswere: daily activities like housekeeping $(n=15,78.9 \%)$; physical activity as a means of transportationbicycle $(n=7,36.8 \%)$; and physical activity at work $(n=5,26.3 \%)$. The practice of physical exercise (walking or sport) was not frequent in the routine of patients with coronary artery disease $(n=6,31.6 \%)$. There was a significant association between marital status and physical activity. Conclusion: The most frequent physical activities were housekeeping; bicycling for transportation; exercise or recreation; and activities at work. There was a predominance of active individuals performingmoderate activities at home or at work and a significant association between activity level and marital status.
\end{abstract}

\section{DESCRIPTORES}

Health Profile.Physical Activity.Coronarydisease.

1 Enfermeira. Universidade Federal do Maranhão. Maranhão. São Luis. Brasil.

2 Enfermeira. Professora Adjunto. Universidade Federal do Maranhão. Maranhão. São Luis. Brasil. 
A atividade física é qualquer movimento corporal produzido pelos músculos esqueléticos que resulta em gasto energético numa ampla variedade de atividades, desde as relacionadas ao trabalho profissional até aquelas consideradas atividades de lazer - caminhadas, jardinagem, trabalhos em casa. Exercício físico é a realização de movimentos corporais produzidos pelos músculos esqueléticos que levam a um gasto energético que envolve intencionalidade de movimento, atividades planejadas, repetitivas e estruturadas, e, desde que a intensidade, a duração e a frequência dos movimentos apresentam algum progresso, ambos, atividade física/exercício físico demonstram igual relação positiva com os índices de aptidão física ${ }^{1}$.

Seja através do incremento da atividade física do seu dia-dia ou da prática regular de exercícios físicos ou do envolvimento em esportes, a atividade física poderá ser suficiente para provocar benefícios no indivíduo. Aatividade física do cotidiano pode, eventualmente, provocar adaptações positivas no condicionamento físico, assim como algumas ocupações profissionais, de tarefas domésticas específicas ou outras atividades cotidianas, pelo seu envolvimento quanto à demanda energética, podem repercutir favoravelmente na aptidão física. A prática regular de exercício físico aeróbico possui um efeito protetor contra a doença cardíaca, entretanto, estudos têm demonstrado que para oferecer esse efeito o exercício tem que ser iniciado precocemente e de maneira permanente ${ }^{2,3,4}$.

Há séculos são reconhecidas as vantagens da atividade física regular, entretanto, apenas na segunda metade do século XXé que programas de reabilitação cardiovascular começaram a ser implantados ${ }^{5}$. Os fatores de risco para a doença coronariana, no decorrer destas últimas décadas, estão aumentando na população mundial, sendo decorrentes especialmente de um estilo de vida sedentário. Um dos maiores benefícios da atividade física regular é a melhora do perfil lipídico, em longo prazo. Estudos demonstram que a atividade física pode auxiliar na prevenção do processo aterogênico contribuindo para o aumento das concentrações de lipoproteína de alta densidade (HDL-C) que têm efeito antioxidante e diminuição dos níveis séricos de lipoproteína de baixa densidade (LDL-C) $)^{5,6}$. O exercício aeróbico promove a diminuição do colesterol total e o aumento da HDL-C ${ }^{7}$.

O tipo de exercício que mais atua no metabolismo de lipoproteínas é o aeróbio, porém, exercícios de força e flexibilidade também são recomendados. A atividade aeróbica exerce função primordial na prevenção e reabilitação, reduzindo sintomas cardiovasculares ${ }^{4}$. Sugere-se a influência positiva da atividade física sobre as alterações morfológicas do coração e artérias coronárias, uma vez que se observa melhora da função cardíaca e do remodelamento cardíaco com redução de placas ateroscleróticas naqueles indivíduos submetidos à reabilitação cardíaca após um evento de infarto ${ }^{5}$. 0 envolvimento efetivo de pacientes coronariopatas ambulatoriais em qualquer tipo de atividade física representa um desafio para a equipe multidisciplinar na reabilitação cardiovascular ${ }^{8}$. Apesar do número de complicações durante programas de reabilitação cardiovascular ser baixo, para a prescrição segura de uma atividade física para pacientes coronariopatas deve-se priorizar recursos materiais adequados, profissionais capacitados, a individualidade do sujeito, atentando-se para a intensidade, frequência, duração e o tipo de exercício ${ }^{9}$. A atividade física realizada sem supervisão, ou seja, com intensidade inadequada pode ocasionar benefícios limitados e provocar efeitos adversos, aumentando o risco ortopédico e especialmente o coronariano ${ }^{10}$.

A atividade física tem sido incorporada como uma conduta terapêutica no tratamento do paciente coronariopata, associado ao tratamento medicamentoso e às modificações de hábitos alimentares e comportamentais. Os efeitos estão diretamente relacionados com o nível da atividade praticada, havendo associação inversa entre o nível de atividade física e o risco de doença coronariana. $A$ atividade de intensidade moderada tem papel importante na saúde e bem-estar de indivíduos saudáveis e também naqueles com eventos cardíacos prévios ${ }^{11}$. Entretanto, observa-se certa resistência à prática de uma atividade física regular, até mesmo em pacientes com um evento cardíaco préexistente, justificado por falta de motivação, falta de tempo e dificuldade de acesso ${ }^{12}$. O objetivo deste estudo foi verificar a prevalência da atividade física em pacientes coronariopatas.

\section{MATERIAL E MÉTODOS}

Trata-se de um estudo transversal, de caráter descritivo realizado em pacientes coronariopata sem um hospital do nordeste brasileiro. Os critérios de inclusão foram pacientes que estavam no pós-operatório mediato de revascularização do miocárdio. Excluíram-se pacientes com dificuldades na fala, distúrbios 
mentais e submetidos à cirurgia de revascularização miocárdica pela segunda vez. Investigaram-se os dados sociodemográficos (idade, sexo, estado conjugal, escolaridade e renda), estilo de vida (dieta, tabagismo, etilismo; hipercolesterolemia, diabetes, obesidade) e medidas antropométricas (peso, altura, índice de massa corporal, pressão arterial e circunferência abdominal), conforme diretrizes ${ }^{13,14}$.

O nível de atividade física foi avaliado pelo Questionário Internacional de Atividade Física - IPAQ (versão longa), conforme se segue: muito ativo e ativo: aquele que cumpriu as recomendações de atividade física; irregularmente ativo: aquele que realiza atividade física, porém insuficientemente para ser classificado como ativo, sendo subdividido em irregularmente ativo $A$ : aquele que atinge pelo menos um dos critérios da recomendação quanto à frequência ou quanto à duração da atividade (frequência: 5 dias/semana ou duração: $150 \mathrm{~min} /$ semana); irregularmente ativo $B$ : aquele que não atingiu nenhum dos critérios da recomendação quanto à frequência nem quanto à duração; sedentário: aquele que não reportou ter realizado nenhuma atividade física por pelo menos 10 minutos contínuos durante a semana ${ }^{15}$.

A coleta de dados ocorreu entre os meses de outubro a dezembro de 2014. Os dados foram digitados no Programa EPI-INFO, versão 7.0 (CDCAtlanta-EUA) e transportados para o programa estatístico IBM SPSSStatistics20 (2011). Realizouse a estatística descritiva e a estimativa de médias e desvio-padrão das variáveis e para verificar associação entre as variáveis foi utilizada a regressão logística univariada para todas as variáveis e regressão logística multivariada para as variáveis que apresentaram o valor de $p<0,20$. Aplicou-se o teste qui-quadrado de independência $\left(\chi^{2}\right)$ entre o nível de atividades e as variáveis sociodemográficas e os fatores de risco. Em todos os testes considerou-se como estatisticamente significante quando o valor de $p$ foi menor ou igual a 0,05 e/ou intervalo de confiança não incluiu o 1. A pesquisa foi aprovada pelo Comitê de Ética em Pesquisado Hospital Universitário da Universidade Federal do Maranhão, tendo recebido parecer favorável $n^{\circ}$ 836.272 em 17 de outubro de 2014.

\section{RESULTADOS}

Houve predomínio do sexo masculino, média etária 50 anos, união estável, pardos, escolaridade baixa, lavradores, renda inferior a dois salários mínimos. Para os fatores de risco cardiovascular predominaram obesidade5 $(26,4 \%)$, hipercolesterolemia 12 (63,2\%), hipertensão arterial $15(78,9 \%)$, diabetes $11(57,9 \%)$, hábitos tabagista e etilista (10,5\%).

A avaliação da atividade física foi caracterizada quanto ao nível, modalidade, intensidade, frequência, duração e tempo gasto sentado. A classificação da modalidade de atividade física -IPAQ, avaliou a caminhada e as atividades diárias (cuidar da casa, uso da bicicleta como meio de transporte) e não somente a prática de exercícios físicos programados. Os pacientes foram classificados por ordem decrescente como ativos $10(53,0 \%)$, irregularmente ativos $A-4(21,0 \%)$, muito ativos - $4(21,0 \%)$ e irregularmente ativos B 1 (5,0\%). O sedentarismo não foi identificado.

A Tabela 1 refere-se a distribuição dos participantes segundo a modalidade da atividade física.

As modalidades de atividades mais frequentes foram cuidar da casa, transportebicicleta, exercício ou recreação e no trabalho (Tabela 1), com uma frequência de seis a sete dias durante a semana com duração de 1 hora (Figuras 1 e 2).

\begin{tabular}{|c|c|c|c|c|c|c|c|c|}
\hline \multirow[t]{2}{*}{$\begin{array}{l}\text { MODALIDADE DA } \\
\text { ATIVIDADE FÍSICA }\end{array}$} & \multicolumn{2}{|c|}{ No trabalho } & \multicolumn{2}{|c|}{$\begin{array}{l}\text { Como meio de } \\
\text { transporte }\end{array}$} & \multicolumn{2}{|c|}{ Em casa } & \multicolumn{2}{|c|}{$\begin{array}{c}\text { Como exercício } \\
\text { ou recreacão }\end{array}$} \\
\hline & $\mathrm{n}$ & $\%$ & $\mathbf{n}$ & $\%$ & $\mathbf{n}$ & $\%$ & n & $\%$ \\
\hline Não & 14 & 73,7 & 12 & 63,2 & 4 & 21,1 & 13 & 68,4 \\
\hline Sim & 5 & 26.3 & 7 & 36.8 & 15 & 78.9 & 6 & 31.6 \\
\hline Total & 19 & 100 & 19 & 100 & 19 & 100 & 19 & 100 \\
\hline
\end{tabular}




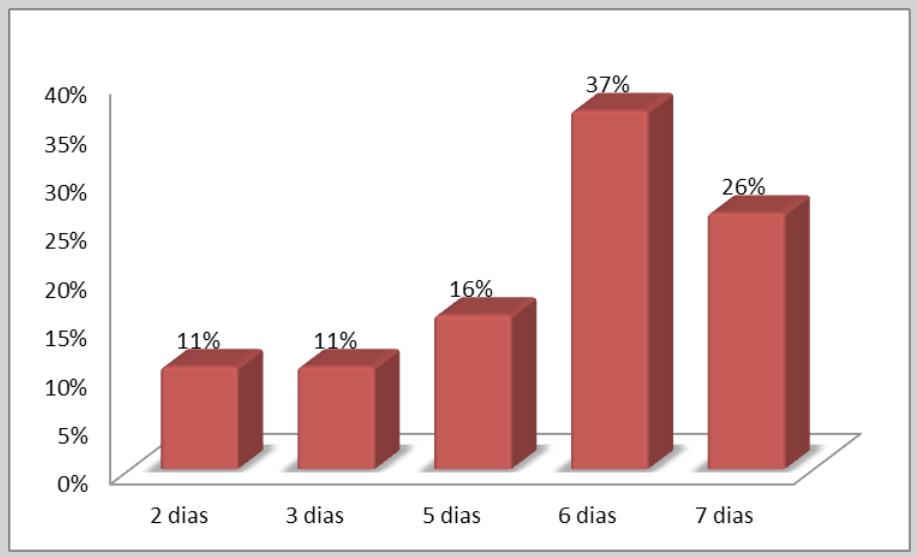

Figura 1. Frequência da atividade física de pacientes coronariopatas do Hospital Universitário da UFMA. São Luís - MA, 2014.

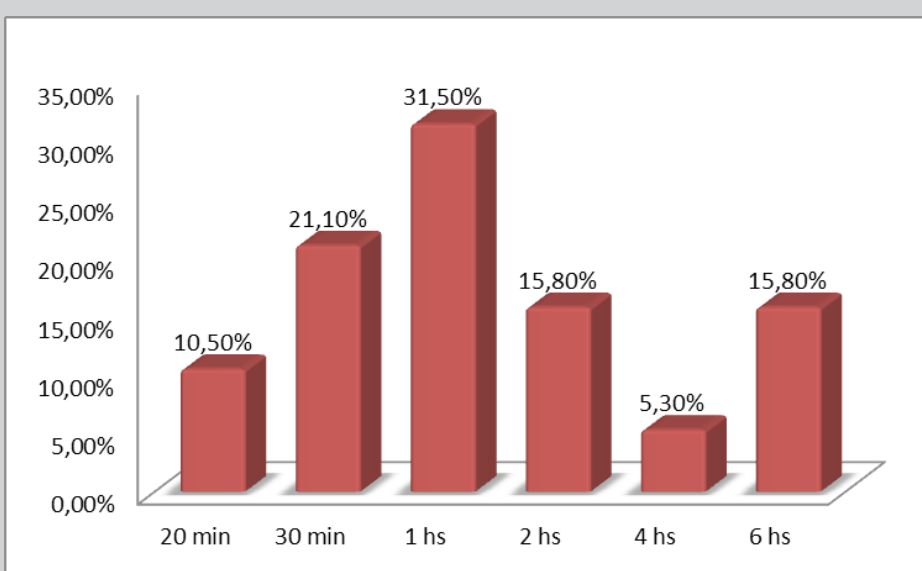

Figura 2. Duração da atividade física de pacientes coronariopatas do Hospital Universitário da UFMA. São Luís - MA, 2014.

A realização da atividade física como exercício físico (caminhada) foi descrita por 6 $(31,6 \%)$ participantes. Em relação à intensidade da atividade física 15 (78,9\%) indivíduos descreveram a realização de atividades moderadas e apenas 4 $(21,1 \%)$ a realização de atividades vigorosas. Não houve relato de atividades consideradas leves. Todos negaram a prática de atividade física moderada.

As proporções entre as variáveis foram comparadas pelo teste qui-quadrado de independência $\left(\chi^{2}\right)$ entre o nível de atividades, as variáveis sociodemográficas e os fatores de risco. Foi encontrada uma associação significativa ( $p<$ $0,05)$ do nível de atividade com o estado civil do paciente, a maioria dos solteiros $(62,5 \%)$ e dos viúvos $(75,0 \%)$ eram ativos e muito ativos, enquanto que os casados e divorciados se apresentaram como ativos $(66,7 \%)$ e irregularmente ativos $(33,3 \%)$, respectivamente (Tabela 2 ).
Na análise univariada de todas as variáveis sociodemográficas e das variáveis consideradas prováveis fatores de risco, não foi encontrada associação significativa $(p>0,05)$.

\section{DISCUSSÃO}

A associação entre os fatores de risco cardiovascular tem efeito cumulativo; isto é, quanto maior o número e intensidade dos fatores de risco, maior a incidência da coronariopatia. Para a promoção da saúde e prevenção da doença coronariana, a atividade física deve ser praticada com a frequência mínima de cinco vezes por semana e trinta minutos de duração para atividades moderadas, e três vezes por semana e vinte minutos para as atividades vigorosas, conforme recomenda o CDC/ASCM (Centro de Controle de Doenças/Colégio Americano de Medicina Esportiva) $)^{11,12}$. 


\begin{tabular}{|c|c|c|c|c|c|c|c|c|}
\hline \multirow[t]{2}{*}{ Variável } & \multicolumn{5}{|c|}{ Nível de Atividade } & \multirow[b]{2}{*}{$\%$} & \multirow[b]{2}{*}{ Total } & \multirow[t]{2}{*}{$p$} \\
\hline & Irregularmente ativo & $\%$ & Ativo & $\%$ & Muito ativo & & & \\
\hline \multicolumn{9}{|l|}{ Sexo } \\
\hline Feminino & 0 & 0,0 & 3 & 100,0 & 0 & 0,0 & 3 & \multirow[t]{2}{*}{0,201} \\
\hline Masculino & 5 & 31,3 & 7 & 43,8 & 4 & 25,0 & 16 & \\
\hline \multicolumn{9}{|l|}{ Faixa etária } \\
\hline $50-54$ & 1 & 33,3 & 1 & 33,3 & 1 & 33,3 & 3 & \multirow[t]{5}{*}{0,261} \\
\hline $55-59$ & 0 & 0,0 & 3 & 75,0 & 1 & 25,0 & 4 & \\
\hline $60-64$ & 3 & 75,0 & 1 & 25,0 & 0 & 0,0 & 4 & \\
\hline $65-69$ & 1 & 50,0 & 1 & 50,0 & 0 & 0,0 & 2 & \\
\hline$>69$ & 0 & 0,0 & 4 & 66,7 & 2 & 33,3 & 6 & \\
\hline \multicolumn{9}{|l|}{ Estado civil } \\
\hline Solteiro & 0 & 0,0 & 5 & 62,5 & 3 & 37,5 & 8 & \multirow[t]{4}{*}{0,010} \\
\hline Casado & 4 & 100,0 & 0 & 0,0 & 0 & 0,0 & 4 & \\
\hline Divorciado & 1 & 33,3 & 2 & 66,7 & 0 & 0,0 & 3 & \\
\hline Viúvo & 0 & 0,0 & 3 & 75,0 & 1 & 25,0 & 4 & \\
\hline \multicolumn{9}{|l|}{ Cor } \\
\hline Branco & 0 & 0,0 & 3 & 75,0 & 1 & 25,0 & 4 & \multirow[t]{3}{*}{0,366} \\
\hline Negro & 1 & 100,0 & 0 & 0,0 & 0 & 0,0 & 1 & \\
\hline Pardo & 4 & 28,6 & 7 & 50,0 & 3 & 21,4 & 14 & \\
\hline \multicolumn{9}{|l|}{ Escolaridade } \\
\hline Analfabeto & 1 & 25,0 & 1 & 25,0 & 2 & 50,0 & 4 & \multirow[t]{6}{*}{0,392} \\
\hline $\begin{array}{l}\text { Fundamental } \\
\text { incompleto }\end{array}$ & 3 & 37,5 & 4 & 50,0 & 1 & 12,5 & 8 & \\
\hline $\begin{array}{l}\text { Fundamental } \\
\text { completo }\end{array}$ & 1 & 100,0 & 0 & 0.0 & 0 & 0,0 & 1 & \\
\hline Médio incompleto & 0 & 0,0 & 2 & 100,0 & 0 & 0,0 & 2 & \\
\hline Médio completo & 0 & 0,0 & 1 & 50,0 & 1 & 50,0 & 2 & \\
\hline Superior completo & 0 & 0,0 & 2 & 100,0 & 0 & 0,0 & 2 & \\
\hline \multicolumn{9}{|l|}{ Procedência } \\
\hline $\begin{array}{l}\text { Município do } \\
\text { Maranhão }\end{array}$ & 1 & 12,5 & 4 & 50,0 & 3 & 37,5 & 8 & \multirow[t]{2}{*}{0,247} \\
\hline São Luís-Capital & 4 & 36,4 & 6 & 54,5 & 1 & 9,1 & 11 & \\
\hline Total & 5 & & 10 & & 4 & & 19 & \\
\hline
\end{tabular}


Em relação às atividades diárias consideradas moderadas, observou-se predomínio de indivíduos ativos. Entretanto, em relação à realização da atividade física como exercício físico (caminhada) a frequência foi menor com intensidade leve. Nenhum dos participantes foi classificado como sedentário. Foi mais freqüente a realização de atividades de seis a sete dias durante a semana.

Os critérios para a classificação das atividades devem considerar no mínimo, a intensidade, frequência e duração da atividade. A característica marcante das atividades desenvolvidas pelos coronariopatas foi traduzida por aquelas realizadas em casa ou no trabalho, apresentando-se como uma atividade de força (mecânica), por serem consideradas atividades vigorosas, que requerem muito esforço. Entretanto, não se pode confundir indivíduos ativos em decorrência de atividades tidas como domésticas, com aqueles considerados ativos pela prática regular de caminhadas, atividades vigorosas ou moderadas, o exercício físico. Muitos participantes do estudo reconheceram não ter esse discernimento, pois acreditavam não haver diferença entre atividade física e exercício físico.

A atividade física, com inclusão de exercícios respiratórios, auxilia na adequação dos parâmetros ventilatórios, com melhora no grau de dispneia, influindo favoravelmente na tolerância ao esforço. A dispneia observada nos pacientes menos graves, sem edema, tem sido mais bem correlacionada ao sedentarismo e anormalidades metabólicas da musculatura esquelética locomotora. Os benefícios ventilatórios observados durante o esforço são parcialmente decorrentes de um retardo no início do acúmulo de lactado sanguíneo, obtido pela elevação nos valores do limiar anaeróbico em testes cardiopulmonares, exibindo menor limitação no desempenho das atividades cotidianas. A atividade física regular tem sido incorporada como uma conduta terapêutica no tratamento do paciente cardíaco, associado ao tratamento medicamentoso e às modificações de hábitos alimentares e comportamentais ${ }^{13,14}$. Pesquisas têm demonstrado que indivíduos que praticam atividade física de moderada a alta intensidade promovem maior proteção para a doença coronariana do que aqueles em atividades realizadas em baixa intensidade ${ }^{14,15,16}$.

Em relação à associação entre atividade física e estado civil, evidenciou-se uma variável protetora para indivíduos solteiros e viúvos que se apresentaram ativos e muito ativos. Uma possível explicação é de que a motivação para a atividade física parecer não estar relacionada somente ao aspecto individual, ambiental e social, mas também ao relacional e emocional-afetivo. Ressalta-se, entretanto, como um fator limitante não ter sido realizado um acom-panhamento longitudinal para descrição mais criteriosa dos aspectos motivacionais que influenciam na atividade física.

Alguns coronariopatas relataram dificuldades em estimar o tempo gasto sentado e afirmaram não conseguir realizar as modificações nos hábitos comportamentais que a patologia requer, considerando as mudanças fastidiosas e difíceis. A intensidade da atividade física deverá sempre ser individualizada e progredir gradualmente, em particular nos pacientes com acentuada intolerância ao exercício. Os períodos de aquecimento (préexercício) e resfriamento (pós-exercício) devem ser mais prolongados, em média quinze minutos e dez minutos respectivamente, principalmente para observação de possíveis arritmias.Aumentos gradativos do nível, intensidade, frequência e duração do tipo de atividade física devem ser sugeridos visando proporcionar uma maior proteção coronariana. Uma poderosa estratégia para prevenir o agravo da coronariopatia deve ter como prioridade o controle dos fatores de risco cardiovascular e o incentivo aos programas de reabilitação cardíaca, o que se configura como uma auditoria da qualidade da atenção à saúde, especialmente quando se pretende oferecer subsídios aos gestores e aos profissionais de saúde na redução do índice de morbimortalidade ${ }^{16,17}$.

Contata-se a dificuldade em encontrar pesquisas que avaliem todos os domínios dos IPAC, ou que avalie atividade física realizada de forma habitual, pois a utilização de instrumentos e critérios distintos inviabiliza uma comparação. Os benefícios 
clínicos da atividade física programada para os coronariopatas torna-se uma evidência, por trazer melhora clínica, hemodinâmica, eletrocardiográfica e metabólica, relacionadas principalmente a redução do colesterol, da sintomatologia cardíaca, do nível de estresse, das alterações musculoesqueléticas e do mau condicionamento físico, devendo, por isso ser estimulada. A adoção de um estilo de vida ativo fisicamente deve fazer parte da rotina diária de indivíduos coronariopatas, buscando uma maior longevidade e qualidade de vida ${ }^{3,17}$.

A prevalência encontrada para fatores de risco considerados modificáveis reflete a necessidade de um enfoque maior na prevenção primária e secundária dos indivíduos, de modo a evitar e/ou controlar eventos coronarianos. Discutese assim, a necessidade de estimular a prática de atividade física regular devidamente orientada por profissionais da área.

\section{REFERÊNCIAS}

1. Milani M, Papa V, Gallo J. Reabilitação Cardiovascular. Manual de condutas clínicas cardiológicas. São Paulo: Segmento Farma, 2005; 2 (1): 287-98.

2. Almeida AML. Efeitos do exercício físico sobre alguns fatores de risco da doença arterial coronariana em pacientes de um centro de reabilitação cardiopulmonar e metabólico. Monografia (Curso de Educação Física) Universidade Federal de Santa Catarina. Florianópolis. 2012.

3. Hélio JCC, Pitanga FJGP Práticas investigativas em atividade física e saúde. Salvador EDUFBA, 2013. 207 p.

4. Agarwal SK. Cardiovascular benefits of exercise. Int J Gen Med.2012; 5 (2): 541-5.

5. Siervuli MTF, Silva AS; Silva AC, Muzzi RAL, Santos ABS. Infarto do Miocárdio: Alterações Morfológicas e Breve Abordagem da Influência do Exercício Físico.RevBrasCardiol.,2014; 27 (5): 349-555.

6. Torres GKV, Marques IR. Estudo sobre o perfil dos pacientes portadores de Síndrome Coronariana Aguda. RevEnferm UNISA. 2012; 13(1): 21-6.

7. American Heart Association. Heart Disease and Stroke Statistics-2012 Update: a report from the American Heart Association. Circulation. 2002; 125(2): 202-20.

\section{CONCLUSÃO}

Houve predomínio de indivíduos ativos nas atividades consideradas habituais moderadas em casa ou no trabalho, contudo, a prática regular de exercício físico (caminhada ou prática desportiva) não foi frequente, assim como da atividade exercício físico e atividades vigorosas. O sedentarismo não foi identificado.

As modalidades de atividades mais frequentes foram cuidar da casa, transportebicicleta, exercício ou recreação e no trabalho, com uma frequência de seis a sete dias durante a semana e duração de 1 hora.

Não houve relato de atividades consideradas leves ou moderadas. Foi encontrada uma associação significativa do nível de atividade e o estado civil.
8. Negrão CE, Barreto ACP. Cardiologia do Exercício: do atleta ao cardiopata. Zerbini: Ciência e Humanismo, 2010.

9. Ribeiro PRQ; Oliveira DM de. Reabilitação cardiovascular, doença arterial coronariana e infarto agudo do miocárdio: efeitos do exercício físico.Revista Digital, 2011; 15(152).

10. FortiM,ZamunérAR, Kunz VC, Salviati MR, Nery TAG, Silva E. Percepção subjetiva do esforço no limiar anaeróbio em pacientes com doença arterial coronariana. Fisioter Pesq.; 2014, 21 (2): 113-119.

11. Haskell WL. Physical activity and public health: updated recommendation for adults from the American College of Sports Medicine and the American Heart Association. Circulation; 2007, 116 (1):1081-93.

12. EirasSB, Silva WHA, Souza DL, VendruscoloR. Fatores de adesão e manutenção da prática de atividade física por parte de idosos. Rev. Bras. Cienc. Esporte; 2010, 31(2):75-89.

13. Sociedade Brasileira de Cardiologia. VI Diretrizes Brasileiras de Hipertensão. ArqBrasCardiol. [Internet]. 2010; 95 (1): 1-51. [acesso em 4ago 2014].

14. Sociedade Brasileira de Cardiologia. V Diretrizes brasileiras sobre dislipidemias e diretriz de prevenção da aterosclerose do departamento de aterosclerose da SBC. ArqBrasCardiol. 2013; 101(4):1-22. 
15. Agarwal SK. Cardiovascular benefits of exercise. Int J Gen Med; 2012, 5 (1): 541-45.

16. Pires RN. Avaliação da correlação da prática da atividade física e qualidade de vida entre pacientes com diagnóstico de insuficiência coronariana. Belém. [Monografia- Graduação]. Universidade da Amazônia; 2010

17. Pinho RA de, Araújo MC, Ghisi GLM, Benetti M. Doença arterial coronariana, exercício físico e estresse oxidativo. ArqBrasCardiol. 2010; 94 (4): 549-55.

\section{Correspondência}

Nome: Líscia Divana Carvalho Silva

Endereço: Av. dos Portugueses, 1966. Bairro: Vila Bacanga, São Luís. Universidade Federal do Maranhão. Departamento de Enfermagem.

CEP: $65080-805$

São Luís - MA - Brasil.

E-mail: liscia@elointernet.com.br 